International Journal for Crime, Justice and Social Democracy

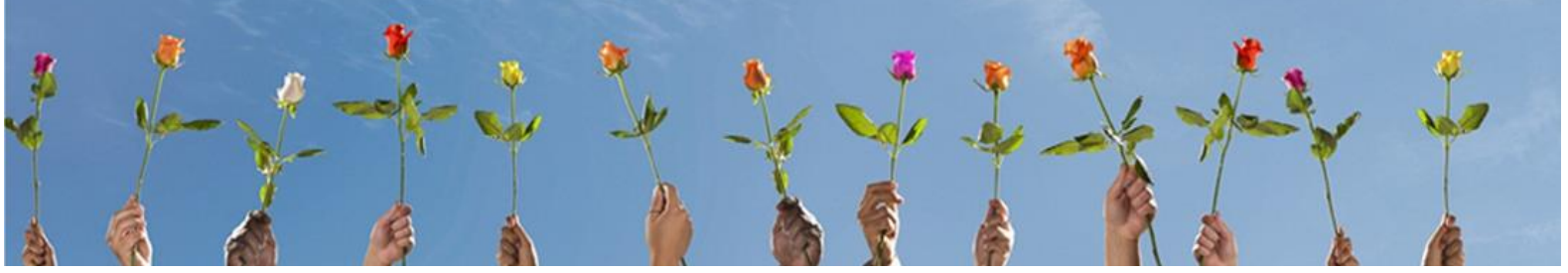

\title{
Primatology, Green Criminology, and the Impacts of Science on the Non-Human World: A Debate from Japan ${ }^{1}$
}

\section{Kazutaka Hirose}

Japan

\begin{abstract}
Primatology was initiated in Japan in 1948 by Kinji Imanishi and his colleagues. A distinctive feature of Japanese primatology is adopting the technique of 'anthropomorphising' nonhuman primates and establishing friendly relationships with them through feeding and other means. Following the anthropomorphic stance of primatology in Japan, yielding many scientific findings, succeeding generations turned to 'biocentrism', which holds that all life, including humans, has equal value. While biocentric values emerged, researchers were also faced with the conflict of having to euthanise wild hybrids per legal mandates to maintain the Japanese macaque (Macaca fuscata) ecosystem. This article analyses the ethical conflicts in Japanese primatology throughout history.
\end{abstract}

\section{Keywords}

Anthropocentrism; anthropomorphism; Japan; non-human primates; speciesism.

Please cite this article as:

Hirose K (2022) Primatology, green criminology, and the impacts of science on the non-human world: A debate from Japan. International Journal for Crime, Justice and Social Democracy. 11(1): 44-57. https://doi.org/10.5204/ijcisd.2016

Except where otherwise noted, content in this journal is licensed under a Creative Commons Attribution 4.0 International Licence. As an open access journal, articles are free to use with proper attribution. ISSN: 2202-8005 


\section{Introduction}

In today's scientific world, it has become ethically problematic for scientists to harm or kill non-human animals for human-centred research. Such a phenomenon (called speciesism), involving prioritising human beings over other species (Beirne 1999; Sollund 2008), has begun to be challenged. Speciesism can be understood as follows: 'Speciesism generally refers to differences in treatment of individual beings on the grounds of their belonging to different biological categories: species in this case' (Noske 1997: 183). One type of animal abuse by humans is animal experimentation in medical research (Regan 2012; Rollin 2007; Sollund 2008), such as harming monkeys in biomedical experiments (Goyes and Sollund 2018).

One researcher points out that humans cannot escape anthropocentrism defined as humanity as 'the measure of all things' (Noske 1997: 183), whether we value or demean non-human animals.

By anthropomorphising non-human primates (i.e., ascribing them human characteristics), Japanese primatology has been able to find various habits often overlooked by simply considering the animal as different from humans. At the same time, the statements and actions of Japanese primatologists have revealed the difficulty of treating non-human animals without falling into speciesism - prejudice against and oppressing animals of other species. They also face difficulty breaking away from anthropocentrism by using the human point of view to interpret the world.

Primatology in Japan began to develop in the 1950s with Kinji Imanishi, Junichiro Itani and Masao Kawai, who observed Japanese macaque monkeys in places such as Koshima Island (Itani 1991: 44-45). Imanishi emphasised the importance of observing from the standpoint of 'anthropomorphism' when attempting to understand the society of Japanese macaque monkeys (Imanishi 1972[1951]). The philosophy of the founding group of researchers who saw humans and other animals as a continuum is evident from the below:

Even human beings themselves were not born outside of nature, but out of nature. They are something that has evolved. Regarding nature, the nature that is the subject of physics is quite different from the nature that is the subject of biology. Even within the nature looked at by biology, the nature of bacteria and amoeba is very different than that of birds and other animals. If we represent nature only as birds and animals then nature may no longer be seen as antipodean in the minds of human beings. (Imanishi 1972[1951]: 145)

Japanese primatologists placing great importance on feeding and identifying individuals based on anthropomorphism reported that a particular troop of Japanese macaque monkeys acquired the habit of washing sweet potatoes given to them as food (Kawai 1965). Subsequent generations studied the 'minds' of captive chimpanzees and, in the absence of a clear legal framework in Japan, moved to establish sanctuaries to protect chimpanzees used for invasive research for medical research purposes (Morimura et al. 2011). Primate conservation efforts were also undertaken.

In recent years, the United States has restricted medical experimentation by assigning 'personhood' to chimpanzees (Bennett and Panicker 2016). However, the actions of Japanese primatologists, which originated in anthropomorphism, are positioned in a global trend of breaking the boundary between nonhuman primates and humans. As for the behaviour of these primatologists, they have distinguished themselves from speciesism in that they treat non-human primates the same as humans.

However, conflicts among primatologists began in the 1970s when Japanese primatologists used tattooing to identify individual animals (Itani 1991: 101-102). However, I would like to point out that if Japanese monkeys were treated the same way as humans, they would not be captured and tattooed.

As Sollund (2008) stated, biased attitudes based on species differences constitute speciesism. In other words, some actions of Itani and others who adopted anthropomorphism can be classified as speciesism. Primatologists even promoted the euthanasia of Japanese macaque monkey and non-native species 
hybrids in the 2000s in Wakayama Prefecture (Setoguchi 2003). Wakayama Prefecture's measures to euthanise crossbreeds can be considered speciesism.

Japanese primatology held up anthropomorphism as the prevailing methodology. However, when we anthropomorphise a particular species, we must bear in mind the following: 'being human-like means being a member of a society' (Root-Bernstein et al. 2013: 1586). There is no contradiction in that Japanese primatology, which began with anthropomorphism, was informed by speciesism attitudes from the beginning. From the standpoint of speciesism, it is possible to anthropomorphise certain animals for the convenience of humans. However, due to their active participation in social activities, speciesism has become apparent in some aspects beyond the framework of anthropomorphism, leading to reconsidering the relationship between humans and non-human animals.

This paper analyses the process by which Japanese primatology, beginning with the philosophy of anthropomorphism, has come to face conflicts for reflecting speciesism throughout its history. It also shows what can be done despite the inevitable anthropocentrism.

\section{The Texts Left by Japanese Primatologists}

Japanese primatologists have published many books for the general public and articles in specialist journals. Imanishi, Itani, and Kawai, the main researchers involved in founding primatology in Japan, have each published books. In writing my paper, I have focused on these collections and consulted the texts they have left behind. I also consulted the journals in which they published their scientific work. The administrative documents of each prefecture and statements of the Primate Society of Japan were accessed through their websites.

\section{The Beginning of Japanese Primatology}

The study of primates in Japan began in 1948 (Imanishi 1994 [1960]: 285). Imanishi had been exploring and researching Mongolia and the Korean Peninsula before World War II. However, after the war, it became immediately difficult to conduct overseas research. Therefore, he decided to conduct field research in Japan (Imanishi 2007 [1973]). In 1948, Imanishi began observing semi-wild horses at the Toi Misaki Cape near Koshima Island in southwestern Japan with his student Itani and team. They discovered wild Japanese macaque monkeys living in the vicinity throughout their observations and shifted their research interest to these monkeys.

\section{Research Results from the Anthropomorphism of Feeding}

Among the founding group, I start by focusing on Kawai based on an analysis of his paper on the behaviour of Japanese macaque monkeys in Koshima Island and from his book, Nihon zaru no seitai (The Ecology of Japanese Macaque Monkeys), reflecting his views on nature. Kawai reports the 'washing of sweet potatoes' by monkeys, referring to how the monkeys hold the sweet potato and dip it in water to remove the dirt and sand from it. It was first discovered in September 1953 when observing a female monkey named Imo (Kawai 1965). The observation was followed by observing parent-child and brother-sister relationships in the troop (Kawai 1965).

In 1956, a colleague also observed 'wheat washing' behaviour, a method of watering the sand to make it easier for monkeys to pick up wheat from the beach (Kawai 1965). Additionally, Kawai reported that when the observer reached into his pocket for peanuts, the monkeys sat in front of him and bent their elbows on one arm, a gesture Kawai called 'give-me-some' behaviour (Kawai 1965). This was observed in 37 of the 47 monkeys (Kawai 1965).

Kawai described the sweet potato washing and wheat washing behaviours as 'inventive' among the three behaviours (Kawai 1965). The give-me-some behaviour was described as 'a kind of attitude toward man 
born out of friendship and composure on the part of the monkeys' (Kawai 1965: 24). The significance of these behaviours in the wake of the feeding was explained by Kawai as follows:

They were given food through provisionization by men, their greatest enemy, and they became to have a friendly relationship with men. That is, a new, different life environment or niche was introduced to their natural life. Therefore, provisionized monkeys suffered changes in their attitude and value system and were given foundations on which pre-cultural phenomena developed. (Kawai 1965: 27)

The term 'provisionisation' used by Kawai here is almost identical to the term 'feeding'. 2 The term 'precultural phenomena' is used by Kawai to avoid an anthropocentric view that only human beings are special creatures capable of having their own culture by deliberately using the word 'culture' while leaving room for differences with human culture.

Kawai struggled with the use of the word 'culture'. In his book on the ecology of the Japanese macaque monkeys, which he wrote for the general public, he used the word 'culture' in katakana instead of 'preculture' (Kawai 1997 [1969]). In Japanese, the word 'culture' is officially written in Chinese characters. However, Kawai used 'katakana' to describe the culture of the Japanese macaque monkey, an uncommon way of using Japanese. Here, we can see the relationship with Imanishi's anthropomorphism, attempting to view humans and non-human animals as a continuous entity. It can be said that by forcing himself to use the word 'culture', Kawai was careful to emphasise the continuity between Japanese macaque monkeys and humans rather than their differences.

Kawai lists four research methods: feeding, identification, long-term observations, and the 'empathy method' (Kawai 1997 [1969]: 308-313).

As already indicated, feeding is a methodology that allows researchers to feed Japanese macaque monkeys to observe their behaviour up close. If some interference is essential to observe the Japanese macaque monkeys, feeding should be recognised as an experimental method (Kawai 1997 [1969]: 308-309). Kawai and his colleagues named and identified the monkeys in the troop and continued to observe them for over 10 years (Kawai 1997 [1969]: 309-310).

On the other hand, Kawai does not explain how the 'empathy method' works clearly. Kawai emphasises the significance of feeling the emotion of the Japanese macaque monkey by joining the crowd. Kawai pointed out that humans are violently rejected by monkeys in the mountains where they live, and even if they could get along with the monkeys, "The very fact that an alien animal, a human being, has entered into a group of monkeys is already disturbing nature' (Kawai 1997 [1969]: 308).

Kawai describes his experience of struggling to identify individual monkeys in Japan. Around the same time as the founders' research on Koshima Island, Kawai first attempted to identify a troop of Japanese macaque monkeys at another location. However, initially, he could not distinguish them at all. However, it became easier to recognise the faces of individual monkeys after three days (Kawai 1997 [1969]: 311). Kawai compared identifying monkeys to how humans remember faces. He said, 'It is the same in the case of monkeys. When you are among a group of monkeys and you adopt their atmosphere, you can recognize them just by looking at their face or figure. The important thing is to experience the atmosphere of the group with your own body' (Kawai 1997 [1969]: 312).

He further explained, 'It is impossible to explain continuous observations of the Japanese macaque simply as that of a cold relationship between observer and object, or as the result of perseverance and effort. It is necessary to have a view of nature that positions this as fate, with a blood relation flowing at its root' (Kawai 1997 [1969]: 314). This is why he emphasised the need to study the Japanese macaque monkey by anthropomorphising it. However, simultaneously, Kawai viewed the relationship between Japanese macaque monkeys and humans as analogous to a 'blood relationship'. In this paper, I later discuss how 
Kawai, a person from Japan, perceived Japanese macaque monkeys as 'blood relatives', which can be observed as a form of nationalism.

In contrast, Itani, a colleague of Kawai, had ambivalent feelings about anthropomorphism. Around the same time as his field research on Koshima Island, Itani was also surveying a troop of Japanese macaque monkeys at Takasakiyama, located approximately $300 \mathrm{~km}$ north of Koshima Island (Itani 2007 [1971]: 46). He wrote about his observation of the monkeys at Takasakiyama: 'To see things as they are, not anthropomorphically, and to find in doing so the nature, habits, and way of life of the primates themselves' (Itani 2007 [1971]: 190-191).

In a later interview with journalist Tachibana, he admitted there had been critics of anthropomorphism. However, he said, 'When we recognize the individuality of primates, we are obligated to view them as anthropomorphic. Recognizing individuality means recognizing the object as having "personality", and personality refers to an individual's character' (Tachibana 1996: 414).

The meaning of 'feeding' was examined by Sugiyama et al. (2013) in a review article. For example, in Takasakiyama, the population of macaque monkeys increased rapidly due to the large amounts of feeding in the 1950s and 1960s initiated by the local mayor. Itani (2007 [1971]) stated that a monk at a local temple began feeding wild Japanese macaque monkeys in the vicinity at the initiative of the mayor of Oita City. The reason behind this was that while Japanese macaque monkeys were popular with tourists, local residents said they were destroying the fields where they were growing their crops (Itani 2007 [1971]). By feeding them, they hoped to attract more tourists and reduce the damage to their fields (Itani 2007 [1971]). It also enabled researchers to observe the feedings of Japanese macaque monkeys. However, the feedings have been controlled since 1965 due to the rapid increase in the macaque monkey population (Sugiyama et al. 2013).

Sugiyama and colleagues acknowledged the significance of feeding. However, they stated that 'Over time, the problems posed by feeding became apparent, and it became a worldwide trend for field researchers to avoid using fed populations as subjects in their studies' (Sugiyama et al. 2013: 75).

\section{Researchers Nursing a Chimpanzee}

As part of anthropomorphism, feeding has become less popular. However, the anthropomorphism espoused by the founding group of Japanese primatology has been passed on to at least some of the researchers of succeeding generations, such as Tetsurō Matsuzawa, ${ }^{3}$ whose work on captive chimpanzees has been published in the field of comparative cognitive science. Matsuzawa emphasised the unity of the four genera of hominids — chimpanzees, gorillas, orangutans and humans (Matsuzawa 2018: 11-12).

In this regard, anthropomorphism, which projects humans onto other animals, continues to have an influence. This may be termed 'biocentrism', which values humans and other living beings equally. Anthropomorphism can be said to have led Matsuzawa to shift his approach to biocentrism. Biocentrism, in this case, 'views human beings as simply "another species" to be attributed the same moral worth as such organisms as, for example, whales,wolves,and birds' (White and Heckenberg 2014: 65 [emphasis in original]). Certainly, chimpanzees and other non-human primates can be considered to have the same moral worth.

Matsuzawa has been observing chimpanzees in captivity for over 40 years at the Primate Research Institute Kyoto University (KUPRI). One of which he named 'Ai' (Matsuzawa 2018: iii). When Ai arrived at the KUPRI, chimpanzees were being used to study hepatitis, and Ai was one of the chimpanzees imported to Japan from Africa (Matsuzawa 2018: 19).

He emphasised the surprise he felt when he first made eye-to-eye contact with the chimpanzee (Matsuzawa 2011: 1). He recalled how this contrasted monkeys' inability to make eye contact (Matsuzawa 2011: 1), stating the following: 'The day I first met her, I realised that this was not a monkey. I can stare at 
her eye-to-eye, and she spontaneously imitates. And there is something about it that touches my heart' (Matsuzawa 2011: 2).

Matsuzawa's experiment showed that baby chimpanzees were better than adult chimpanzees, and even human adults, with their 'working memory of a number among many numbers instantly flashed on a screen' (Inoue and Matsuzawa 2007).

Matsuzawa described the significance of this experiment as follows: 'This was a break with the sterile dichotomy of 'human and animal' and the anthropocentric worldview' (Matsuzawa 2018: 156 [emphasis in original]). According to Matsuzawa, it was known that chimpanzees could understand human language and use tools to some extent (Matsuzawa 2018: 156), but that was only a part of what humans can do (Matsuzawa 2018: 156). However, this numeric memory task demonstrated that chimpanzees could do what humans could not and better (Matsuzawa 2018: 156).

Matsuzawa and his colleagues also reported on the care of chimpanzees by the staff after they had been disabled by disease (Hayashi et al. 2013). One chimpanzee was born in captivity at the KUPRI and was found lying motionless on the ground at 24 . He was diagnosed with the human disease of acute transverse myelitis (Miyabe-Nishiwaki et al. 2010). They published a paper documenting 41 months of human care for a chimpanzee who was temporarily paralysed from the neck down (Hayashi et al. 2013). The work of Matsuzawa et al. can be considered an extension of the 'empathy method' described by Kawai. This is because the researcher observed the chimpanzees' cognitive abilities, and the people and chimpanzees were actively involved in the caregiving and taking process. The continued involvement of humans in rehabilitating chimpanzees is at least different from the position that chimpanzees should only be subjected to human-demanded experiments.

In his book, which includes illustrations of the rehabilitation of chimpanzees, Matsuzawa emphasised the progress in analysing the genetic information of living organisms. He said, "The idea that "all living things are always connected" is a new view of humanity established in the 21st century. All living things must have been connected at some point in time. At that point, they were the same single creature' (Matsuzawa 2018: 3 [emphasis in original]). Matsuzawa (2018: 11) went on to say, 'Humans are in no way special beings. All forms of life are unique and special'.

\section{Animal Protection Among Japanese Primatologists}

The paper has clarified the process of developing Japanese primatology, from anthropomorphism to biocentrism. Japanese primatology generated new knowledge about primates. It also became increasingly involved in primate conservation efforts. For example, Matsuzawa has worked to ban the use of chimpanzees for biomedical research (Morimura et al. 2011). He and his colleagues established the Chimpanzee Sanctuary Uto (CSU) in April 2007 in Japan (Morimura et al. 2011), which became the first sanctuary for retired laboratory chimpanzees in Japan. According to Morimura et al. (2011), the chimpanzees in the CSU can live in an environment where welfare and environmental enrichment are considered.

This was a big challenge in Japan, a country that lacks a legal system to prohibit invasive experiments on apes (Morimura et al. 2011). An estimated 150 wild chimpanzees were brought to Japan in the 1970s to be used in medical experiments for hepatitis B and C (Morimura et al. 2011). A pharmaceutical company, Sanwa Kagaku Kenkyusho Co Ltd. (Sanwa), the forerunner of CSU, started breeding seven chimpanzees in 1978 (Morimura et al. 2011). The breeding led to an increase in the chimpanzee population, and Sanwa conducted medical research on hepatitis $C$ and malaria in the 1990s (Morimura et al. 2011). In 2000, they had 117 chimpanzees in captivity (Morimura et al. 2011).

Meanwhile, researchers and zoo officials, including Matsuzawa, founded the Support for African/Asianbased Great Apes (SAGA) in 1998 (Morimura et al. 2011; SAGA's website is https://www.sagajp.org/indexe.html). 'SAGA was based on three main principles: (1) to assist in the conservation of great 
apes and their natural habitats; (2) to enhance the quality of life of great apes in captivity; and (3) to prevent the use of great apes as subjects in invasive studies, and to promote their scientific understanding through noninvasive techniques' (Morimura et al. 2011: 227).

At the urging of SAGA and the Japanese Primatological Society, a committee including chimpanzee researchers was formed at Sanwa in 1998 (Morimura et al. 2011). Following these activities, invasive medical experiments on chimpanzees at Sanwa came to an end in 2006 (Morimura et al. 2011). The CSU was established in 2007 (Morimura et al. 2011), with Sanwa responsible for maintaining the facility and KUPRI responsible for its operation. Members of SAGA and others created a facility to protect chimpanzees from invasive experimentation in a different framework than the law while seeking the cooperation of corporations.

The actions of Matsuzawa and his colleagues in denying access to chimpanzees in developing cures for human diseases can be described as biocentrism, not speciesism, in that they treat humans and chimpanzees as equals. After over 50 years of development, its scope has expanded beyond the confines of science to include social primate conservation efforts.

\section{The Conflicts Facing Japanese Primatology}

I analysed the process by which Japanese primatology, characterised by anthropomorphism, evolved into biocentrism and related activities to protect non-human primates. However, despite their anthropomorphic approach, Japanese primatologists have also 'harmed' non-human primates at times. The fact that they emphasised the continuity between human and non-human animals while also harming the latter is a major paradox.

In the following section, I would like to discuss speciesism in Japanese primatology by examining the following two cases: tattooing Japanese macaque monkeys and exterminating non-native and hybrid species to protect the macaque ecosystem.

\section{Tattoos on Japanese Macaque Monkeys}

In identifying and observing individual monkeys and their behaviour, Kawai stated that individuals could be identified by empathy rather than by distinct features, much in the same way as humans can be identified. However, Itani's behaviour at Takasakiyama was not always ideal. Itani initially identified macaques at Takasakiyama in the same way as Kawai did and was able to remember about 300 individuals (Itani 1991: 97-100).

After that, however, the number of individuals at Takasakiyama continued to increase. Reflecting on 1972, Itani (1991: 101) said, 'Becoming overwhelmed by the ever-increasing number of monkeys, I was forced to use tattoos'. Regarding the specific approach, he states the following:

We caught the monkeys in box traps, anesthetized them, put them to sleep, took biometric measurements as well as their fingerprints, examined their health, and even took blood samples. On the faces of the examined monkeys, we made dots with ink and then lightly tapped them with a bundle of cotton sewing needles. (Itani 1991: 101)

According to Sugiyama and Ohsawa (1988), tattooing began in 1971, and at least 50 to 100 monkeys were tattooed each year from 1971 at Takasakiyama. The tattoos were used to identify individuals and study the trends of a growing troop. Of course, individual identification was necessary for research and conservation purposes. However, this raises questions about the ethics of tattooing monkeys for such a reason.

Cazaux (2012: 103) makes the following statement, from the perspective of green criminology, which is helpful for interpreting the act of tattooing monkeys: 
an anthropocentric-imposed distinction is made between animal abuse and animal use, given the acceptability or necessity of the animal suffering to be overridden with economic or scientific imperatives.

Cazaux words carry a strong critique about the biased perspective from which humans mark the boundaries between legitimate and illegitimate exploitation of animals. From an anti-speciesist perspective, it is unacceptable to capture and injure wild Japanese macaques (or any other animal). It is important, however, to note that Japanese primatologists, who considered humans and monkeys as a continuous entity and who believed that they could be identified by the 'empathy method', used the act of tattooing to identify individuals.

Itani (1991: 103) too did not fully accept tattooing as a research method:

The method of tattooing appears to be solid, rational, and in accordance with the path trodden in modern science. However, the method of identifying specimens by oneself has some advantages that should not be overlooked. It is not solely limited to identification, but draws the researcher into an examination of the individuality of the monkeys. In other words, it makes it possible to presume or even be certain what kind of behavior an individual will or will not exhibit under certain conditions.

Itani and his team's series of trapping, anaesthetising and tattooing Japanese macaque monkeys do not represent an attitude of non-human beings equal to humans. Itani's article shows that he emphasised the 'individual relationship' between monkeys and researchers. At any rate, this is an example of 'speciesism' lurking among researchers of Japanese primatology. As observed in the works of Imanishi and Kawai, Japanese primatology introduced anthropomorphism into the science of observing animals as objective subjects and adopted the method of researching the individual relationship between researchers and individual macaque monkeys. However, the disconnection between monkeys and human researchers has existed since early on.

\section{Euthanasia of Crossbreeds}

Japanese primatologists have taken a position on the crossbreeds of around 2,000 Japanese macaque monkeys and the exotic wild Formosan macaque (Macaca Cyclopis) in Wakayama Prefecture, promoting euthanising crossbreeds and exotic species. One of the organisations that strongly urged local governments to perform euthanasia was the Primate Society of Japan (PSJ). The PSJ was founded in 1985 to promote mutual understandings between different fields, foster primate researchers from a broad perspective, and carry out organisational activities as a group of researchers in various parts of the world. ${ }^{4}$ The society's first president was Kawai (PSJ's website is http://www.primatesociety.com/former staff.html).

In 2001, the society called on the governor of the Wakayama Prefecture, where interbreeding was spreading, promoting the capture and euthanasia of all non-native Formosan macaque monkeys to avoid disturbing the ecosystem (PSJ 2001). PSJ (2001) was concerned that the Japanese macaque monkeys, endemic to Japan, were increasingly becoming hybridised with an introduced species, the Formosan macaque monkeys.

The Wakayama Prefecture began to eradicate the Formosan macaque monkeys and their hybrids and declared the eradication of the species in 2017 (Wakayama Prefecture 2017). The PSJ statement takes the position that, at least in Japan, the Japanese macaque monkey is more important than the Formosan macaque monkey or any other mixed-breed species.

Here, I discuss the humans' perception of their role in ecosystem regulation. I focus on tracing the details of the crossbreed problem in Wakayama Prefecture, an invasive species. Hybridisation between the 
Formosan and Japanese macaques has been a problem outside the Wakayama Prefecture (Kawamoto et al. 2005). However, in the case of Wakayama Prefecture, there was a great deal of controversy involving the local government, as described below.

\section{The Process by which 'Hybrids' Came to Be}

The Wakayama Prefecture reported that 10 to 30 macaque monkeys living in a private tourist facility in the Oike area of the Wakayama Prefecture approximately 50 years ago had somehow become feral (Wakayama Prefecture 2001). In other words, there is a great possibility that the macaques brought in by humans had escaped and became wild in the vicinity. Around 2000, hybridisation between Formosan and Japanese macaque monkeys was discovered in the vicinity of the Oike area, causing damage to the crops (Wakayama Prefecture 2001). A survey showed that the troop was spread over $14 \mathrm{~km}^{2}$ and had a population of about 200 individuals (Wakayama Prefecture 2001).

Although Japanese and Formosan macaques are considered distinct species, they can interbreed, and their hybrids can also breed. The Wakayama Prefecture (2001) reported the results of morphological and genetic surveys, noting that over $60 \%$ of the macaques observed in the Oike area had shorter tails than those of the Formosan macaques. These reports also explained that, after collecting samples from nine macaques, six were interbred with two pure Formosan macaques and one pure Japanese macaque.

\section{Crossbreed Eradication Program}

The Wakayama Prefecture (2001) announced a policy of capturing all Formosan macaques and hybrids living in the Oike area. Japan's Convention on Biological Diversity and the National Biodiversity Strategy explained that the capture of macaques is necessary to prevent a disturbance of the ecosystem by invasive species (Wakayama Prefecture 2001). Although it is possible to enclose the habitat of macaques and hybrids with electric fences, it is technically and financially challenging to capture them. The Wakayama Prefecture chose to euthanise them after capturing them in cages (Wakayama Prefecture 2001). Aside from the captive Formosan macaques and hybrids, the Japanese macaques and residents whose crops had been damaged were also 'victims' of the escape of the Formosan macaques from the entertainment facilities nearly 50 years ago (Wakayama Prefecture 2001). Notably, the Wakayama Prefecture described the Formosan and Japanese macaques as 'victims'.

Given this scenario, the Wakayama Prefecture started to exterminate the macaques and their hybrids, although this decision involved a complicated process. As it was difficult to obtain the documents leading up to implementing the plan in the Wakayama Prefecture, I refer to a 2003 article by Akihisa Setoguchi on the process.

According to the article, the prefectural government was required to consult a prefectural council before this plan was drawn up. This council disagreed with the euthanasia plan and reported that it would consider a survival plan (Setoguchi 2003). Consequently, the Wakayama Prefecture decided to consider alternatives, such as releasing the captured monkeys to uninhabited islands. By April 2001, the Wakayama Prefecture had received 241 comments across Japan, most of which protested the eradication project.

\section{Primate Society of Japan (PSJ) Statement and Residents' Survey}

However, in response to these developments, the PSJ and other stakeholders issued an opinion piece strongly calling for euthanasia (Setoguchi 2003). The PSJ (2001) issued a letter of request to the governor of Wakayama Prefecture on 18 April 2001, emphasising that the Japanese macaque is an endemic species and strongly urging the euthanasia of the crossbreed species. The society expressed concern about the progress and spread of hybridisation of the Formosan macaque. The letter also pointed out that releasing the monkeys to uninhabited islands could affect the islands' ecosystems, and keeping them in huge cages would also be problematic from animal welfare and escape prevention perspectives. The following was requested: 'Based on the conclusion of the Wakayama Prefecture Monkey Conservation and Management 
Plan Study Group, we hope that Wakayama Prefecture will promptly start capturing monkeys based on a policy that all monkeys should be captured and euthanized in principle' (PSJ 2001). The letter also added that the PSJ would spare no effort to help realise this request.

On 20 April 2001, the Wakayama Prefecture surveyed 1,000 residents (Setoguchi 2003). The questionnaire asked participants to choose between euthanising all captured monkeys or keeping them in zoos. The results indicated that $69.9 \%$ of the respondents supported the euthanasia plan. In September 2001, the Wakayama plan was officially implemented. However, Setoguchi (2003: 125-126) stated the following:

It should be noted that the question asked in the questionnaire was about the treatment of the monkeys after their removal and was based on the principle that this removal had taken place. Wakayama Prefecture, in accepting the request of the expert group, rejected alternatives such as releasing the monkeys to uninhabited islands and excluded them from the questionnaire.

Consequently, 'Citizens were left with only two choices: a breeding plan with an initial cost of 1.1 billion yen, or a euthanasia plan that would cost only 1 million yen' (Setoguchi 2003: 126). In other words, Setoguchi's analysis suggested that the Wakayama Prefecture, with the strong backing of experts such as the PSJ, promoted the capture and euthanasia of Formosan macaques and hybrids.

Setoguchi (2003) noted a gap between the perceptions of experts and non-experts regarding concerns about ecological disturbances, such as primatologists. Setoguchi (2003) cited criticism based on values different from those of primate specialists that the stance of protecting the 'pure blood' of Japanese monkeys leads to nationalism. As I explained earlier, Kawai, one of the founding members of the Japanese Primatology Group, used the term 'blood relationship' to describe the relationship between Japanese macaques and researchers.

\section{Nationalism and the Crossbreeding Problem}

The criticism from the perspective of nationalism introduced by Setoguchi was that of Nagao Nishikawa (2001). He was a researcher who examined nation-states and colonialism at that time, citing an article from the Asahi Shimbun newspaper reporting the expected euthanasia of a Japanese macaque and Formosan macaque crossbreed in the Wakayama Prefecture. Nishikawa (2001: 2) stated the following: 'While we are still not free from the horrors of eugenics, something terrible is happening in the name of ecology and ecologism'.

Nishikawa (2001) noted that, around the same time, the Asahi Shimbun published an article about the suffering of patients with Hansen's disease for years due to the Japanese government's misguided isolation policy. The hardships of Hansen's disease patients who had been unfairly treated as non-nationals are superimposed on crossbreeds (Nishikawa 2001). He went on to note that 'It is a fact that nation-states have distinguished between citizens and foreigners by establishing national borders and nationalities, and at the same time, have sought to unify the nation by distinguishing between good citizens and bad citizens, that is, citizens and non-citizens, within the same nation' (Nishikawa 2001: 2-3).

Setoguchi (2003) referred to a paper by Nishikawa (2001), noting that the exclusion of invasive species has historically often been strongly associated with nationalism. For example, the campaign to preserve native flora in Germany under the Nazi regime. He also stated that the Formosan macaque problem (each monkey name included the name of a region) and the expression of eliminating 'miscegenation' to protect the 'pure blood' of the Japanese macaque monkeys easily led to associations with human societies. He speculated that this is also because 'monkeys are closely related to humans, and the fact that a view of animals that anthropomorphizes monkeys is deeply rooted in our culture, is one of the factors that led to the two being associated with one another' (Setoguchi 2003: 130). 


\section{The Complexity of the Crossbreeding Problem}

Setoguchi (2003) noted that invasive species of the Formosan macaque involves a multidimensional set of problems, including nature conservation, animal protection and nationalism. He further stated that the exclusion of non-native species and hybrids to conserve biodiversity does not inevitably lead to nationalism: 'Biodiversity is an idea that aims to protect the local biota in each region and by no means aims to protect national nature' (Setoguchi 2003: 129-130).

Setoguchi (2003) emphasised the importance of 'pluralism' in considering the problem of the Formosan macaque in the Wakayama Prefecture. Regarding invasive species, such as the Formosan macaque, Setoguchi stated, 'It would be desirable to satisfy social rationality by following a process of legitimate consensus-building along pluralistic values' (Setoguchi 2013: 131).

Hybridisation is also a problem in other areas of Japan. Outside of the Wakayama Prefecture and in the Chiba Prefecture, controlling hybrids with rhesus monkeys is an issue (Chiba 2021). Hybridisation occurs naturally in animals and plants during the evolutionary process (e.g., Mallet 2007). However, when humans are involved in hybridisation, as in the case of Formosan macaque monkeys in Wakayama Prefecture, the species are often subject to extermination.

The position of building consensus among a plurality of values appears to be a reasonable conclusion. Notably, however, only humans are assumed to be members of the consensus-building process here. When we prioritise consensus among humans to determine how non-human animals should live, the possibility of speciesism cannot be eradicated.

\section{The Speciesism of Primatologists}

The disconnect between humans and monkeys has been visible since the Japanese macaque monkeys were tattooed. The disconnect became more apparent from the response of primatologists to crossbreeding in the Wakayama Prefecture after 2000.

It can be said that scientists' attitude of observing the Japanese macaques as they are has resulted in speciesism. The notion that racism and sexism are unacceptable is shared, whereas speciesism is not observed as a similar problem (Sollund 2008). Sollund (2008) points out a past history of the exploitation of certain populations based on race and gender in the name of 'science': 'By regarding others as very different, be it based on physical traits as skin colour, brain size or sex, it is possible to legitimate exploitation' (Sollund 2008: 116). Speciesism in scientific research is in danger of making the same mistake.

\section{Conclusion}

In this paper, I traced the progress of Japanese scientists who began to study non-human primates with the anthropomorphism methodology around 1950. The position of the Japanese primatologists has evolved into one that allegedly treats humans and other primates as equals, indicating biocentrism.

Observing the non-human primate using anthropomorphic methods, while scientifically meaningful, is speciesism, given that it places the human's intellectual interests above those of other species. Conversely, from a biocentric viewpoint, 'humans are not superior and all species have an intrinsic value where harm reduction towards animals is preferred above human interests' (van Uhm 2017: 333 [emphasis in original]). Matsuzawa's commitment to biocentrism and the rehabilitation and protection of chimpanzees from medical experimentation is a reasonable course of action. However, simultaneously, they manifested their speciesism by euthanising species deemed by humans as unsuitable to be in Japan by treating them as alien species and crossbreeds. Srinivasan and Kasturirangan (2017) noted that when humans deal with invasive species, they often focus on the maintenance of the ecosystem as a whole rather than the rights 
of each individual species. This alone does not immediately lead to speciesism. However, killing to maintain the ecosystem does.

Piers Beirne (2014: 61) defined human-caused animal death as theriocide, stating:

How and why some theriocides are constructed as socially acceptable and others as unacceptable must surely be problematized as a key object of inquiry. Dare I say it, but a killing is a killing is a killing, no matter whether it is regarded as acceptable or not.

Airenti (2018) pointed out that anthropomorphism is arbitrarily applied to animals with which humans contact. The issue of hybrids epitomises the arbitrary application of anthropomorphism by primatologists. It is necessary to discuss the words and actions of Japanese primatologists from the perspective of speciesism. They could have chosen to break away from the traditional scientific viewpoint of protecting the endemic species of Japanese macaques and not intervene in the crossbreeding that had already started to prevent falling into the trap of speciesism. The problem in the Wakayama Prefecture, where other hybrids were sacrificed to protect the endemic species of non-human animals, illustrates the speciesism embedded in science. As Sollund (2008) noted, the 'science' of craniometry has made women and some races inferior. History has shown that 'being scientific' has contributed to various forms of discrimination (Goyes, 2016).

It can be understood as the actions of human primatologists according to their situational interests. There is no contradiction in sometimes killing crossbreeds or protecting them from being used in other experiments to secure the non-human primate for their research. All of them are still anthropocentric in their treatment of animals in accordance with human values.

Japanese primatology began with an emphasis on continuity with other animals and a methodology of anthropomorphism. I think it is necessary to go beyond this limit by challenging the behaviour of primatologists, which must inevitably be criticised as speciesism. We can never get over anthropocentrism completely-because we inevitably see it as humans-but we can change the situation for the better.

\footnotetext{
1 I have translated Japanese works into English. When the title of the work is in Japanese, the English translation is written in parentheses. When referring to a collection of works or a book, if the exact year of publication of the underlying article is known, it is noted in square brackets [ ].

2 Although there is some debate over the use of technical terms such as provisionisation (Asquith 1989), Kawai (1997 [1969]) uses the term in his Japanese book to refer to the feeding of animals by humans in general

3 In June 2020, Kyoto University announced the results of an investigation that revealed that four Kyoto University primate researchers, including Matsuzawa, had been accused of irregular accounting amounting to about JPY 500 million between fiscal years 2011 and 2014 (Kyoto University 2020 http://www.kyoto-u.ac.jp/ja/about/events news/office/kouseichousa/news/2020/2006261.html (in Japanese)). The accounting irregularities in the maintenance of chimpanzee cages at the Primate Research Institute and the Wildlife Research Center, in my opinion, are of serious concern. However, since this is not a fraudulent accounting of the research results, which Matsuzawa has so far voted for, I have cited his papers and books.

4 The Primate Society of Japan website is https://primate-society.com/index.html
} 


\section{References}

Airenti G (2018) The development of anthropomorphism in interaction: Intersubjectivity, imagination, and theory of mind. Frontiers in Psychology 9: 2136. https://doi.org/10.3389/fpsyg.2018.02136

Asquith PJ (1989) Provisioning and the study of free-ranging primates: History, effects, and prospects. Yearbook of Physical Anthropology 32(S10): 129-158. https://doi.org/10.1002/ajpa.1330320507

Beirne P (1999) For a nonspeciesist criminology: Animal abuse as an object of study. Criminology 37: 117-148. https://doi.org/10.1111/j.1745-9125.1999.tb00481.x

Beirne P (2014) Theriocide: Naming animal killing. International Journal for Crime, Justice and Social Democracy 3(2): 49-66. https://doi.org/10.5204/ijcisd.v3i2.174

Bennett AJ and Panicker S (2016) Broader impacts: International implications and integrative ethical consideration of policy decisions about US chimpanzee research. American Journal of Primatology 78(12): 1282-1303. https://doi.org/10.1002/ajp.22582

Cazaux G (2012) Labelling animals: Non-speciesist criminology and techniques to identify other animals. In Beirne $\mathrm{P}$ and South $\mathrm{N}$ (eds) Issues in green criminology: Confronting harms against environments, humanity and other animals 87-113. New York: Routledge.

Chiba Prefecture (2021) Dai niji Chiba ken akage zaru boujo jisshi keikaku (The second implementation plan for the control of Rhesus monkeys in Chiba Prefecture) (In Japanese).

Goyes DR and Sollund R (2018) Animal abuse, biotechnology and species justice. Theoretical Criminology 22(3): 363-383.https://doi.org/10.1177/1362480618787179

Goyes DR (2016) Green activist criminology and the epistemologies of the south. Critical Criminology 24, 503-518.

Hayashi M, Sakuraba Y, Watanabe S, Kaneko A and Matsuzawa T (2013) Behavioral recovery from tetraparesis in a captive chimpanzee. Primates 54: 237-243. https://doi.org/10.1007/s10329-013-0358-2

Imanishi K (1972) [1951] Ningen izen no shakai(Prehuman Societies).Doubutshu no shakai (The animal society):138-241. Tokyo: Shisaku-Sha (in Japanese).

Imanishi K (1994)[1960] Gorira(Gorilla) In Itani J, Umeyama S, Umesao T, Kawakita J, Kira T, Kuwabara T and Morishita M(eds)Imanjishi Kinji zenshu 7 (Imanishi Kinji collection 7). Tokyo: Kodan-Sha (in Japanese).

Imanishi K (2007) [1973] Watashi no rireki sho (My resume). Watashi no rireki sho:Kagaku no kyudosha (My resume: Science Seekers):13-118. Tokyo: Nihokeizaishimbun Shuppannsha (in Japanese).

Inoue S and Matsuzawa T (2007) Working memory of numerals in chimpanzees. Current Biology 17(23): R1004R1005. https://doi.org/10.1016/i.cub.2007.10.027

Itani J (1991) Saru hito afurika watashi no rireki sho (Monkeys, humans, and Africa my resume). Tokyo: Nihokeizaishimbun-Sha (in Japanese).

Itani J (2007)[1971]Takasakiyama no saru (The Monkeys of Takasakiyama). In Ota I, Sawachika T, Terashima H and Yamagiwa J (eds) Itani Junichiro Chosakushu 1(Itani Junichiro Collection 1):39-262. Tokyo: Heibon-Sha (in Japanese).

Kawai M (1965) Newly-acquired pre-cultural behavior of the natural troop of Japanese monkeys on Koshima islet. Primates 6(1): 1-30. https://doi.org/10.1007/BF01794457

Kawai M (1997) [1969] Nihon zaru no seitai (The ecology of Japanese macaques monkeys). Kawai Masao Chosakushu 2(Kawai Masao Collection 2): 37-320. Tokyo: Shogakukan (in Japanese).

Kawamoto Y, Kawamoto S and Kawai S (2005) Shimokita hanto ni okeru taiwan zaru to nihon zaru no kozatsu(Hybridization of introduced Taiwanese macaques with native Japanese macaques in Shimokita Peninsula, Aomori, Japan). Primate Research 21(1): 11-18 https://doi.org/10.2354/psj.21.11 (in Japanese)

Mallet J (2007) Hybrid speciation. Nature 446: 279-283. https://doi.org/10.1038/nature05706

Matsuzawa T (2011) Souzou suru chikara:Chimpanii ga oshietekureta ningen no kokoro (The power of imagination: What chimpanzees have taught us about the human heart ). Tokyo: Iwanami-shoten (in Japanese).

Matsuzawa T (2018) Wakachiau kokoro no shinka (Evolution of the spirit of sharing). Tokyo: Iwanami-Shoten (in Japanese).

Miyabe-Nishiwaki T, Kaneko A, Nishiwaki K, Watanabe A, Watanabe S, Maeda N, Kumazaki K, Morimoto M, Hirokawa R, Suzuki J, Ito Y, Hayashi M, Tanaka M, Tomonaga M, Matsuzawa T (2010) Tetraparesis resembling acute transverse myelitis in a captive chimpanzee (Pan troglodytes): Long-term care and recovery. Journal of Medical Primatology 39(5): 336-346. https://doi.org/10.1111/j.1600-0684.2010.00415.x

Morimura N, Idani G and Matsuzawa T (2011) The first chimpanzee sanctuary in Japan: An attempt to care for the "surplus" of biomedical research. American Journal of Primatology 73(3): 226-232. https://doi.org/10.1002/ajp.20887

Nishikawa N (2001) Kokuimin to hikokumin no aida aruiha 'minzokujouka' nitsuite (Between nationals and nonnationals, regarding 'ethnic cleansing'). Shiso 927: 1-3 (in Japanese)

Noske B (1997) Speciesism, Anthropocentrism, and non-Western cultures. Anthrozoös 10(4): 183-190.

https://doi.org/10.2752/089279397787000950 
Primate Society of Japan (2001) Taiwan zaru shu-dan no jokyo ni kansuru yobosho (Request for the removal of the Formosan monkey). https://primate-society.com/files/20010418.pdf (in Japanese)

Regan T (2012) Vivisection: The case for abolition. In Beirne P and South $\mathrm{N}$ (eds) Issues in green criminology: Confronting harms against environments, humanity and other animals: 114-139. New York: Routledge.

Rollin BE (2007) Animal research: A moral science. Talking point on the use of animals in scientific research. EMBO Reports 8(6): 521-525. https://doi.org/10.1038/sj.embor.7400996

Root-Bernstein M, Douglas L, Smith A and Veríssimo D (2013) Anthropomorphized species as tools for conservation: Utility beyond prosocial, intelligent and suffering species. Biodiversity and Conservation 22: 15771589. https://doi.org/10.1007/s10531-013-0494-4

Setoguchi A (2003) Inyushu mondai to iu souten (The problem of introduced species). Gendai-Shiso 31(13): 122134 (in Japanese).

Sollund R (2008) Causes for speciesism: Difference, distance and denial. In R. Sollund (Ed.), Global Harms: Ecological Crime and Speciesism (109-131). Oslo: Nova Science Publishers, Inc.

Sugiyama Y and Ohsawa H (1988) Takasakiyama ni seisoku suru eduke nihon zaru kotai gun no doutai to kanri (Population dynamics and management of baited Japanese monkeys at Takasakiyama). Primate Research 4(1): 33-43. https://doi.org/10.2354/psj.4.33 (in Japanese)

Sugiyama Y, Watanabe K, Kurita H and Nakamichi M (2013) Reityourui gaku no hatten ni eduke ga hatashita yakuwari (The role of artificial feeding on the development of primatology). Primate Research 29(2): 63-81. https://doi.org/10.2354/psj.29.011 (in Japanese)

Srinivasan K and Kasturirangan R (2017) Conservation and Invasive Alien Species: Violent Love.In Maher J, Pierpoint $\mathrm{H}$ and Beirne P (eds) The Palgrave International Handbook of Animal Abuse Studies: 433-452. London: Palgrave Macmillan:Tachibana T (1996) 'Sarugaku no genzai ge' (Monkeyology today vol. 2).Tokyo: Bungeishunju(in Japanese)

van Uhm DP (2017) A green criminological perspective on environmental crime: The anthropocentric, ecocentric and biocentric impact of defaunation. Revue Internationale de Droit Pénal 87(1): 323-340.

Wakayama Prefecture (2001) Wakayama ken saru hogo kanri keikaku (Wakayama prefectural monkey conservation management plan). Wakayamakempo(Wakayama Prefectural Gazette) No. 1285 (in Japanese).

Wakayama Prefecture (2017) Oike chiiki no taiwan zaru oyobi nihon zaru to no kouzatu zaru no mure no konzetu ni tsuite (About the eradication of the colony of macaque monkeys crossing with the Formosan and Japanese macaque monkeys in the Oike area) (in Japanese).

White R and Heckenberg D (2014) Green criminology: An introduction to the study of environmental harm. London: Routledge. 